\title{
Functional Approach to Protocols Specification
}

\author{
Jakub Bojanowski ${ }^{\mathbf{a}}$, Michal Iglewski ${ }^{\mathbf{b}}$, Jan Madey ${ }^{\mathbf{a}}$, Abdellatif Obaid ${ }^{\mathbf{b}}$ \\ ${ }^{a}$ Institute of Informatics, Warsaw University, Banacha 2, 02-097 Warsaw, Poland, \\ Email: kuba@mimuw.edu.pl, madey@mimuw.edu.pl \\ b Département d'informatique, Université du Québec à Hull, Hull, Québec, Canada J8X 3X7 \\ Email: iglewski@uqah.uquebec.ca, obaid@uqah.uquebec.ca
}

\begin{abstract}
This paper investigates the applicability of the functional documentation approach, and in particular the trace assertion method, to specification of modern communication systems. As an example a class of communication protocols known as sliding window protocols was chosen.

\section{Introduction}

Documentation methods based on simple mathematical concepts, as advocated in [10], attempt to make computer systems design similar to classical engineering. In the approach called "functional" (since mathematical functions and relations play a key role) we deal with a formal documentation which guarantees the required precision to allow verification and systematic testing. Documents discussed in [10] were meant to cover a variety of applications, including modern communication systems. The hierarchical structuring of those systems, recommended by ISO $[7,8]$, is especially suitable for the functional approach. In particular, the trace assertion method used for module specification, seems to be a good candidate for investigation of its applicability for protocol specification.

Some attempts were already made to use the trace assertion method for specification of protocols $[2,3,5,9]$. In this paper we suggest a certain modification of the method (with respect to input variable events) and present its possible application to a class of protocols known in the literature under the name "sliding window protocols" [8]. These protocols are often used as a case study (e.g. in [4]) and also have been discussed in [3].
\end{abstract}

\section{Informal description of a sliding window protocol}

The concept of sliding window is used within many data-link layer protocols (e.g. HDLC, LAPD, X.25 Level 2). In this study we consider a simplified version of the protocol in which data are sent in one direction only. Hence, we use the term user_sender for the user sending data, and user_receiver for the user receiving them. Data transmitted from user_sender to user_receiver are divided into frames which are sequentially sent by the use of the lower layer services (user_receiver receives frames in the order they were sent by user_sender). Those services are provided by protocol entities; we use the term sender for the entity providing services to user_sender, and receiver for the entity providing services to user_receiver. Sender transmits 
frames received from user_sender to receiver, who sends acknowledgments of those frames to sender, and forwards received frames to user_receiver.

We assume that the communication between protocol entities is unreliable, i.e. any frame (containing data or the acknowledgment) may be lost or delayed. We also assume (for generality) that the initial order of frames may be changed between sender and receiver.

\subsection{Algorithms for protocol entities}

All frames issued by user_sender are sequentially numbered. The frames received by sender are sent to receiver, but their copies are kept in the fixed size sending window. If this buffer is full, then user_sender stops issuing frames. Frames remain stored in the sending window until their arrival to the destination is acknowledged. When the acknowledgment is received, the window slides to the new position. The acknowledgment of a certain frame is also the acknowledgment of all frames within the window with lower sequence numbers.

Since frames may be delivered in any order or even lost, receiver must store them in a buffer, called the receiving window ${ }^{\mathrm{a}}$. A frame may be accepted by receiver only if its sequence number fits in the window; frames whose numbers cannot fit, are ignored. If the received frame and all frames with lower sequence numbers are correctly received, the acknowledgment is sent (through the lower layer) to sender. If more than one frame is pending for acknowledgment, receiver sends the acknowledgment for the frame with the highest sequence number. The acknowledged frames are forwarded to user_receiver, and then the receiving window slides to the new position.

If the acknowledgment of a frame does not reach sender within a certain fixed time period (called the time-out), then all frames kept within the sending window are retransmitted. As a consequence, more than one copy of each frame may reach receiver. In such a case acknowledgements of all copies must be issued by receiver.

\section{Functional documentation}

In this paper we deal with one of the documents needed during the process of computer system design, implementation, and verification, namely with a module interface specification [11], in which the trace assertion method (in short: TAM) is used to describe externally observable behavior of software modules.

\subsection{Trace assertion method}

In general case, a module is understood as a group of visible access-programs using a hidden data structure. A module can be viewed as implementing one or more finite state machines called objects. All communication between the outside world and the objects imple-mented by a given module is achieved via:

(a) a vector of external state variables that the object "observes" (the input variables),

(b) a vector of variables that are controlled by the object and can be observed externally (the output variables), and

(c) the module's access-programs that can be used by other modules to provide information to the object, and/or receive information from it.

The values of the input variables are considered to be observable at any time. To observe them the module may use hardware devices or externally supplied programs. Actual observa-

a. In the rest of this paper we will assume that the receiving window is of size 1 . 
tions are made at points in time determined by the environment.

A state change in the object may be caused only by an external event, i.e. an invocation of an access-program, or a change in the values of the input variables.

A trace is a sequence of events affecting the object. If the event is an access-program invocation, the trace includes also those output values (returned by this access-program) which influence the module's future behavior. The set of traces is partitioned into a finite number of equivalence classes. Each class is represented by one of its elements called the canonical trace. Intuitively, the canonical traces correspond to the states of the module. The set of canonical traces is characterized by the predicate, called canonical.

A complete black-box description of a module in TAM is described by the following:

(a) functions that map from single event extensions of canonical traces to the equivalent canonical traces; these extension functions define the equivalence relation on traces,

(b) a relation that maps each canonical trace to a vector of sets of values of output variables,

(c) relations that associate each single (access-program) event extension with a set of returned values for each output argument.

\subsection{Input variable events}

Output variables were widely used in example specifications and though their role has changed during the evolution of TAM, their semantics is well understood. Input variables, however, were introduced in the report [11] in an intuitive way. The term input variable event was not clearly defined. Its semantics was proposed in further papers $[2,3]$, where events were defined by a relation between old and new values of the input variables. In [2], new values of the input variables were used within the extension function definition, so a canonical trace did not provide complete information about the state of the module.

We propose an alternative semantics for such events. We assume that input variables are discrete and the changes of their values are atomic, mutually exclusive. Therefore, any two of such changes will be sequentially ordered by the module and we do not have to consider events corresponding to changes of more than one variable at a time. An event corresponding to a variable occurs every time when the value of the variable changes. Values of input variables cannot be used directly within the specification. A new value assigned to the variable may be placed as an event argument (read only), and then it may be referred within other sections of the specification. The old value of the input variable, or the values of other input variables, may be obtained from the canonical trace. Every event corresponds to one variable, and every variable has one event related to it, so events may be defined in the same table as input variables are.

\subsection{Application to protocol specifications within the ISO OSI Reference Model}

In the ISO model four types of primitives are recognized: requests, responses, indications, and confirmations. However, this classification is too detailed for our approach. If we consider a certain (N)-entity, then we want to distinguish only: (a) between incoming and outgoing primitives, and (b) between $(\mathrm{N})$-primitives and $(\mathrm{N}-1)$-primitives. In other words, we will recognize the following four classes of primitives: $(\mathrm{N})$-incoming, (N)-outgoing, $(\mathrm{N}-1)$-incoming and $(\mathrm{N}-1)$-outgoing.

The representation of incoming and outgoing primitives must be different. Let us focus on $(\mathrm{N}-1)$-incoming primitives. Two different representations of these primitives are possible in the current version of TAM. Either an access-program list may be extended by access-pro- 
grams provided to the upper layer entities, or input variables may be used. The first solution does not seem to be appropriate. We cannot assume that during the design of the ( $\mathrm{N}-1)$-layer entities, the access-program list and canonical traces of the (N)-layer are known.

If we apply only input variable events, then we can use a single approach for the description of all the layers of a network. Since incoming primitives are described as input variable events, the outgoing primitives must be represented as changes of the output variable values. The variables themselves ${ }^{b}$ represent the ISO service-access-points (SAP's).

In the OSI model, peer entities cooperate together by exchanging protocol-data-units (PDU's) which are placed as service-primitives arguments. Hence, the variables representing SAP's will be defined as variables of PDU types. The modules introducing these types will provide access-programs for coding informations within variables of PDU types.

\section{An example of a protocol specification}

We will illustrate the approach presented above by specifying the receiver entity from the sliding window protocol. Details about the structure, syntax and semantics of a TAM specification is outside the scope of this short paper. We must first, however, give a general overview of the cooperating modules $\mathrm{c}$.

\subsection{Specifications of protocol entities}

A complete description of a protocol requires specifications of protocol entities, and specifications of PDU. For the purpose of verification, a formal specification of the lower layer is also needed. In this paper we deal only with the specifications of protocol entities. Lower layer entities and PDU's are not formally specified, though it should not be too difficult to do so. The presented approach is suitable for any layer of the network, thus creating specifications of lower layer entities is straightforward. PDU's are classical data structures and there are many examples of applications of TAM to such structures (cf. e.g. [6, 11]).

The receiver entity is specified as one module (called Receiver); the sender entity for sake of simplicity is split into two modules (called Sender and Timeout_Ctrl). The module Sender is responsible for sending and receiving PDU's; the module Timeout_Ctrl, only for reporting time-out events.

Dependences between entities are shown on the Fig. 1. As mentioned before, modules cooperate by the use of input/output variables only (denoted by arrows). An input variable in one module is an output variable in the other. Changes of variable values represent service primitives, so the names of the values correspond to the appropriate primitive types. The types $\left(<\mathrm{N} \_\mathrm{PDU}\right\rangle,<\mathrm{N}+1$ _PDU $>$ ) represent types of protocol-data-units. For the sake of simplicity, we decided to use only one PDU type ( $<$ N_PDU $>$ ) for both data frames and acknowledgment frames. Such a solution is also suitable for the general case of the sliding window protocol, where acknowledgments are sent together with data frames. The value denoted by the empty trace of the <N_PDU > type is distinguished - it represents the "empty message". We assume that setting such a value to the output variables of $<$ N_PDU $>$ type (i.e. N-1_RES_SAP and N -1 _REQ_SAP) does not change the state of the modules in which those variables appear as the input ones.

b. Note, that a variable appears as the input variable in one module, and as the output variable in the other.

c. A more complete example, in which these modules are also specified, is to be found in the technical reports published by the author's universities. 


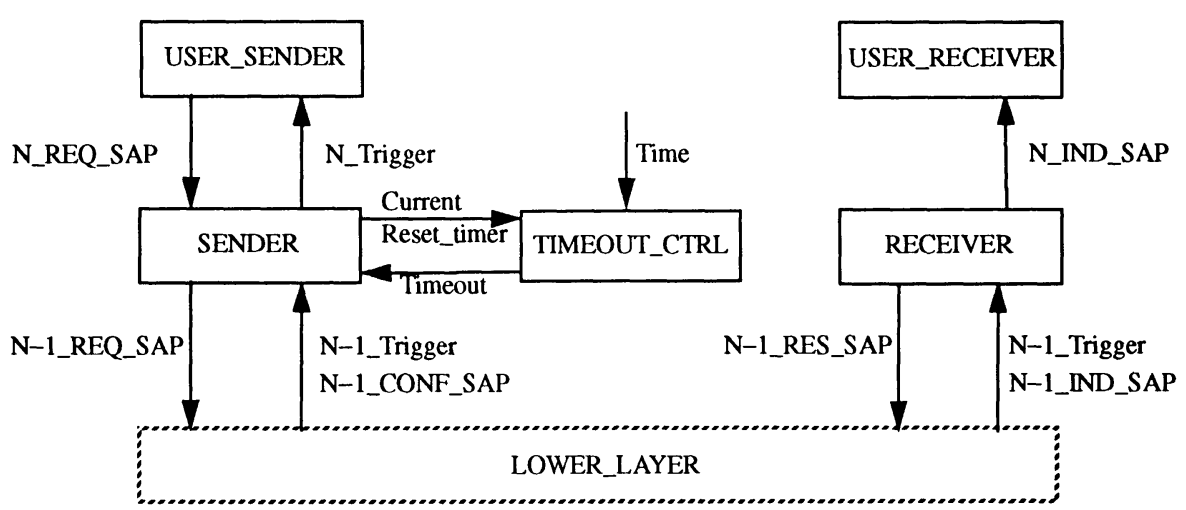

Figure 1. Dependencies between entities.

\subsection{Receiver Module Interface Specification}

\section{Informal description}

This specification describes a protocol entity receiving frames according to the sliding window protocol. Receiving window size is 1 . The module contains no access-programs, but only input and output variables.

\section{INPUT VARIABLES}

N-1_IND_SAP - N_PDU delivered by the lower layer entity.

$\mathrm{N}-1$ - Trigger - the variable changes its value when the lower layer is ready to receive another $\mathrm{N}$-service-primitive.

\section{OUTPUT VARIABLES}

N_IND_SAP - the N+1_PDU currently being sent to the higher layer.

N-1_RES_SAP - the N_PDU (acknowledgment) currently being sent to the lower layer.

\section{CANONICAL TRACES}

$$
\operatorname{IND}(s, m) \cdot\left[\text { ACCEPTED] }{ }_{1}^{\mathrm{d}}\right.
$$

The IND occurrence corresponds to the highest sequence number of all frames received by the entity. If ACCEPTED occurs in the trace, the receiving window is empty, and the entity is ready to accept another frame.

\section{OTHER ASSUMPTIONS}

The specified module receives frames of type $<$ N_PDU $>$ from the lower layer, and sends frames of type $<\mathrm{N}+1$ _PDU $>$ to the user. We assume that the module $<$ N_PDU $>$ includes the access-programs CODE (translating tuples (<integer $>,\left\langle N+1 \_P D U\right\rangle$, <integer $>$ ) to the type $<$ N_PDU $>$ ) and DECODE (translating N_PDU's back to the tuples). 


\section{(0) CHARACTERISTICS}

- type specified: <receiver>

- features: single-object, deterministic, non-parameterized

- foreign types: $<$ natural $>\stackrel{\text { df }}{=}\{\mathrm{i}:<$ integer $>\mid \mathrm{i} \geq 0\},\langle$ flipflop $>\stackrel{\text { df }}{=}<$ boolean $\rangle,\langle$ N_PDU $>,<N+1$ PDU $>$

\section{(1) SYNTAX}

\section{INPUT VARIABLES}

\begin{tabular}{|c|c|c|}
\hline Variable name: & Type: & Event: \\
\hline N-1_IND_SAP & $<$ N_PDU $>$ & IND $(s, m)$ where $(s, m, a)=$ N_PDU::DECODE(N-1_IND_SAP) \\
\hline N-1_Trigger & <flipflop $>$ & ACCEPTED \\
\hline
\end{tabular}

\section{OUTPUT VARIABLES}

\begin{tabular}{|c|c|}
\hline Variable name: & Type: \\
\hline N_IND_SAP & $<$ N+1_PDU $>$ \\
\hline N-1_RES_SAP & $<$ N_PDU $>$ \\
\hline
\end{tabular}

\section{(2) CANONICAL TRACES}

$\operatorname{canonical}(\mathrm{T}) \Leftrightarrow \exists \mathrm{s}, \mathrm{m}|\exists \mathrm{d}:\{0 . .1\}| \mathrm{T}=\mathrm{IND}(\mathrm{s}, \mathrm{m}) .\left[\right.$ ACCEPTED] ${ }_{1}^{\mathrm{d}}$

(3) EQUIVALENCES

_ $\equiv \mathrm{IND}(0, \mathrm{~N}+1$ _PDU::_).ACCEPTED

T.ACCEPTED $(\mathrm{s}, \mathrm{m}) \equiv>$

\begin{tabular}{|c|c|}
\hline Condition & Equivalence \\
\hline$\exists \mathrm{S} \mid \mathrm{T}=\mathrm{S}$.ACCEPTED & \%already_accepted $\%$ \\
\hline$\forall \mathrm{S} \mid \mathrm{T} \neq \mathrm{S}$.ACCEPTED & T.ACCEPTED \\
\hline
\end{tabular}

T.IND $(s, m) \equiv$

\begin{tabular}{|c|c|c|}
\hline \multicolumn{2}{|c|}{ Condition } & Equivalence \\
\hline \multirow{2}{|c|}{$\forall \mathrm{S} \mid \mathrm{T} \neq \mathrm{S}$.ACCEPTED } & \%last_not_accepted\% \\
\hline \multirow{3}{*}{$\exists \mathrm{s}_{1}, \mathrm{~m}_{1} \mid \mathrm{T}=\mathrm{IND}\left(\mathrm{s}_{1}, \mathrm{~m}_{1}\right)$.ACCEPTED } & $\mathrm{s}<\mathrm{s}_{1}+1$ & $\mathrm{IND}\left(\mathrm{s}_{1}, \mathrm{~m}_{1}\right)$ \\
\cline { 2 - 3 } & $\mathrm{s}=\mathrm{s}_{1}+1$ & IND $(\mathrm{s}, \mathrm{m})$ \\
\cline { 2 - 4 } & $\mathrm{s}>\mathrm{s}_{1}+1$ & $\mathrm{IND}\left(\mathrm{s}_{1}, \mathrm{~m}_{1}\right)$.ACCEPTED \\
\hline
\end{tabular}




\section{(4) RETURN VALUES}

\section{OUTPUT VALUES}

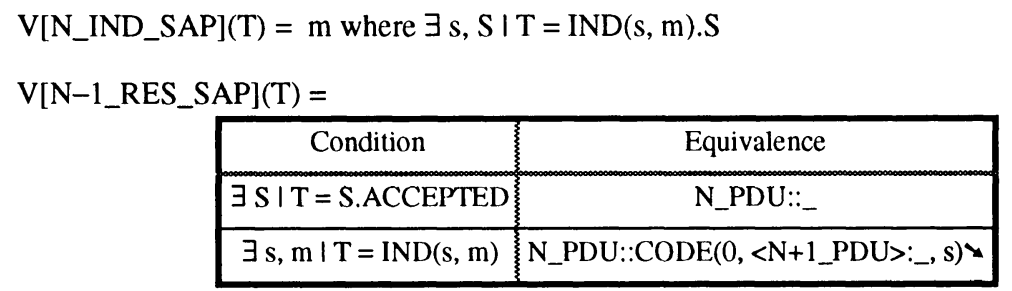

\section{Conclusions}

\subsection{Related works}

The first application of TAM to protocols was proposed about 10 years ago by Hoffman [5] but he used an early version of the method [1] and some of his results are now obsolete. One of the conclusions of Hoffman's work was that access-programs were not sufficient to define the behavior of protocol entities. He introduced output access-programs to express the way modules influence the environment but this concept was not introduced in further versions of the method (it was replaced by the notion of the output variables).

After the TAM report [11] was published, Parnas proposed a general framework for using functional approach in protocol specifications [9]. He suggested to divide a specification of a layer into a service specification - describing the syntax and the semantics of services, and a protocol design - describing services implementation. The disadvantage of this proposal is that the TAM specifications of a layer (service specifications) would not describe all externally observable behaviors of the layer, but only its interface to the higher one. Using the OSI model terminology we may say that in this approach the specifications describe only (N)-primitives; a method for expressing $(\mathrm{N}-1)$-primitives was not given.

A different approach was presented in [3]. A layer was not decomposed into entities but a protocol specification was given from the external observer point of view. To simplify canonical traces, a large number of auxiliary functions was introduced, and the specification was split into three modules. The hierarchy of the modules did not correspond to the layered structure of network but the proposed approach may be useful during the protocol verification. The input variables were used to describe a cooperation with the timer.

In the recent paper [2] by Courtois and Parnas, the approach suggested in [9] was not used. This work presented a specification of the MAC protocol used within FDDI network. The specification of the protocol entity was complete - interfaces to both higher and lower layers were defined. Input and output variables were widely used, but semantics of input variable events was different from the one introduced in the current paper. Since the interface to the higher layer was described by the use of access-programs, the proposed approach cannot be applied to specify more than one layer of a network.

\subsection{Applicability of the functional documentation}

After some minor changes (proposed in this paper) the trace assertion method seems to be quite suitable to the protocol specifications. Specifications are relatively short and understand- 
able (once one got accustomed to the formalism). Basic OSI model concepts, such as SAP and service primitives, are reflected in the specifications. The main advantage of this approach is that the same formalism is used for specifications of protocol entities and for specifications of data structures (PDU's); other known to us methods do not have this property.

\section{Acknowledgements}

This paper was inspired first of all by works of D.L. Parnas and his coauthors. We are very grateful to him for his guidance. M. Kubica and K. Stencel offered us many helpful comments on earlier versions of the paper.

This work was partly supported by the Natural Sciences and Engineering Research Council of Canada, by the State Committee for Scientific Research in Poland, and by Digital Equipment's European External Research Programme.

\section{References}

1 Bartussek, W., Parnas, D.L., "Using Traces To Write Abstract Specifications For Software Modules", Proceedings of 2nd Conference of European Cooperation in Informatics, Lecture Notes in Computer Science, 65. Springer-Verlag, Venice, 1978.

2 Courtois, F., Parnas, D.L., "Formally Specifying A Communications Protocol Using The Trace Assertion Method", CRL Report No. 269, McMaster University, CRL, Telecommunications Research Institute of Ontario (TRIO), Hamilton, Ontario, Canada, 1993.

3 Desrosiers, B., Iglewski, M., Obaid, A.,"Utilisation de la méthode des traces pour la définition formelle d'un protocole de communication", Proceedings of CFIP'93: Colloque Francophone sur L'Ingénierie des Protocoles, Montreal, Quebec, Canada, 1993.

4 Hogrefe, D., "OSI formal specification case study: the Inres protocol and service, revised", IAM-91-012, Universität Bern, Institut für Informatik, Bern, Switzerland, May 1991; Update May 1992.

5 Hoffman, D., "The Trace Specification of Communication Protocols", IEEE Transactions on Computers, Vol. C-34, No. 12, December 1985, pp. 1102-1113.

6 Iglewski, M., Madey, J., Parnas, D.L., Kelly, P.C. "Documentation Paradigms". $C R L R e-$ port No. 270, McMaster University, CRL, Telecommunications Research Institute of Ontario (TRIO), Hamilton, Ontario, Canada, 1993.

7 ISO 7498, International Standard Organization, OSI - Basic Reference Model, International Standards Organization, 1984.

8 Jain, B.N., Agrawala, A.K., Open Systems Interconnection: Its Architecture and Protocols, Elesevier, 1990.

9 Parnas, D.L., "Documentation of Communications Services and Protocols", Technical Report 90-272, Queen's University, C\&IS, Telecommunications Research Institute of Ontario (TRIO), Kingston, Ontario, Canada, 1990.

10 Parnas, D.L., Madey, J., "Documentation of Real-Time Requirements”, in: Kavi, K.M. (editor), Real-Time Systems. Abstraction, Languages, and Design Methodologies, IEEE Computer Society Press, 1992, pp. 48-56.

11 Parnas, D.L., Wang, Y., "The Trace Assertion Method of Module Interface Specification”, Technical Report 89-261, Queen's University, C\&IS, Telecommunications Research Institute of Ontario (TRIO), Kingston, Ontario, Canada, 1989. 